\title{
A study of the low-temperature oxidation of a long chain aldehyde: $n$-hexanal
}

\author{
Anne Rodriguez, Olivier Herbinet, Frédérique Battin-Leclerc \\ Laboratoire Réactions et Génie des Procédés, CNRS-Université de Lorraine, 1 rue Grandville, \\ 54000 Nancy, France
}

Published in the Proceedings of the Combustion Institute (2017),36, 365-372

doi:10.1016/j.proci.2016.05.047

\begin{abstract}
Large aldehydes are important species among the oxygenated products formed during the oxidation of fuels (e.g. alkanes or alkenes) and biofuels (e.g. long chain alcohols), but their oxidation chemistry has been scarcely studied up to now. In this work, a study of the oxidation of hexanal has been performed in a jet-stirred reactor over the temperature range 475-1100 K, at a residence time of $2 \mathrm{~s}$, a pressure of $106.7 \mathrm{kPa}$, an inlet fuel mole fraction of 0.005 and at three equivalence ratios $(0.25,1$ and 2). Reaction products were quantified using two analytical methods: gas chromatography and cw-cavity ring-down spectroscopy. In addition to species usually measured during oxidation study (such as $\mathrm{CO}, \mathrm{CO}_{2}$, water, $\mathrm{H}_{2} \mathrm{O}_{2}$ and $\mathrm{C}_{1}-\mathrm{C}_{3}$ olefins, aldehydes and ketones), specific low temperature oxidation species were also observed: $\mathrm{C}_{6}$ species, such as $\delta$ - and $\gamma$-caprolactones and hexanoic acid, but also $\mathrm{C}_{5}$ species, such as 2-methyltetrahydrofuran and pentanal which are typical reaction products detected during the oxidation of $n$-pentane. A detailed kinetic model has been developed using an automatic generation software. This model was used to highlight the specificities of the oxidation chemistry of long chain aldehydes and to understand the formation routes of the $\mathrm{C}_{5}$ and $\mathrm{C}_{6}$ low-temperature oxidation products. Simulations indicate that the low energy of the aldehyde $\mathrm{C}-\mathrm{H}$ bond has a significant effect on the fuel reactivity and on the distribution of reaction products.
\end{abstract}

Keywords: Oxidation; Aldehyde; $n$-Hexanal; Jet-stirred reactor; Modeling 


\section{Introduction}

The oxidation chemistry of long chain aldehydes (defined here as species with more than two carbon atoms) is of utmost importance because they are typical intermediates formed during the oxidation of conventional fuels and biofuels. As an example, the formation of important amount of long chain aldehydes was observed during the low-temperature oxidation of olefins via the Waddington mechanism [1], as well as during the low-temperature oxidation of long chain alcohols [2]. To a lesser extent aldehydes are also typical low-temperature oxidation products from alkanes. Note also that new types of fuels, such as bio-oil, produced from biomass pyrolysis contains many molecules which also include aldehyde functions [3]. Yet there are still very few data in the literature about aldehyde oxidation chemistry, especially at low-temperature. To our knowledge, there is no data available for aldehydes larger than $n$-pentanal.

A literature review shows that the studies of the oxidation and pyrolysis of propanal, $n$-butanal and $n$-pentanal were mainly performed using shock tubes [4-8] or burners [9] in the high temperature region. To our knowledge, the only low-temperature oxidation experiments were carried out by Veloo et al. in a flow tube $[10,11]$ over the temperature range $500-1200 \mathrm{~K}$. The species investigated were propanal, $n$ - and iso-butanal. The kinetic analysis of the model at 655 $\mathrm{K}$ showed that propanal was mainly consumed by $\mathrm{H}$-atom abstraction involving the $\mathrm{H}$-atom of the aldehyde group forming a radical which decomposed to $\mathrm{CO}$ and an ethyl radical through $\alpha$ scission [10]. The oxidation of the ethyl radical was then responsible for the low-temperature reactivity of propanal. A larger low-temperature reactivity was observed for $n$-butanal than for propanal and no low-temperature reactivity for iso-butanal [11]. According to a kinetic analysis performed at $645 \mathrm{~K}, n$-butanal was mainly consumed via $\mathrm{H}$-atom abstraction involving the $\mathrm{H}$ atom of the aldehyde group (65\% of the consumption of the fuel). This radical reacted in two ways: via an $\alpha$-scission to $\mathrm{CO}$ and --propyl radical (30\%) and an addition to $\mathrm{O}_{2}$ forming a peroxy radical (70\%). This peroxy radical and the $n$-propyl radical can undergo low-temperature oxidation chemistry providing the formation of branching agents through the formation of peroxy-butanoic acid and $n$-propyl-hydroperoxide. No specific low-temperature oxidation products such as cyclic ethers with the same skeleton as the fuel were detected during these studies.

The goal of the present work was to investigate the oxidation chemistry of a larger aldehyde, $n$-hexanal, in a jet-stirred reactor in order to better understand the oxidation chemistry of this type of reactant and to highlight the possible effect of the chain length on the reactivity and the nature of reaction products.

\section{Experimental section}

The experimental apparatus and analytical techniques used in the present work have already been described in previous papers and only the main features are discussed in the manuscript (a more detailed description is given as supplementary material).

Experiments were carried out in a fused silica jet-stirred reactor. It is a continuous stirred tank reactor operated at steady state. It was designed to have homogeneous concentrations and temperatures for residence times between 0.5 and $5 \mathrm{~s}$ [12]. The homogeneity is ensured by turbulent jets exiting four nozzles located in the center of the spherical reactor. The reactor is 
preceded by an annular preheater (made of two concentric tubes) which helps maintain a homogeneous temperature. Both preheater and reactor were heated up to the reaction temperature using Thermocoax resistances with temperature control using type $\mathrm{K}$ thermocouples. The reaction temperature was measured with an independent type $\mathrm{K}$ thermocouple located in the intra annular part of the preheater with the extremity close to the four nozzles in the center of the reactor (temperature uncertainty of $\pm 5 \mathrm{~K}$ ). The liquid fuel flow rate was controlled using a Coriolis flow controller, mixed with the carrier gas (helium) and evaporated in a heat exchanger. Oxygen flow was added at the inlet of the reactor to minimize possible reactions with the fuel before entering the reactor. Helium and oxygen flow rates were controlled using mass flow controllers. The relative uncertainty in flow rates were $\pm 0.5 \%$ according to the manufacturer, which resulted in a relative uncertainty of about $\pm 0.5 \%$ in the residence time of the gas in the reactor. The fuel was provided by Sigma-Aldrich (purity > 98\%). Gases (oxygen and helium) were provided by Messer (purities of 99.995\% and 99.999\%, respectively).

Two diagnostics were used to quantify the reaction products. Most of the species were analyzed using gas chromatography (GC) with a flame ionization detector (FID) for the detection of carbon containing species. The use of a methanizer (hydrogenation on nickel catalyst) enabled the quantification of $\mathrm{CO}$ and $\mathrm{CO}_{2}$. Formaldehyde was also detected by GC but the quantification was difficult and not precise as the peak had a long tail and a retention time close to that of other species (methanol, acetaldehyde and oxirane). Relative uncertainties in mole fractions were estimated to be $\pm 5 \%$ for species which were calibrated using standards and $\pm 10 \%$ for species which were calibrated using the effective carbon number method and for species whose peaks are co-eluted (as formaldehyde).

Formaldehyde, as well as water and hydrogen peroxide, were quantified using a spectroscopic technique: continuous wave cavity ring-down spectroscopy. This technique relies on the absorption of light (in the near infrared range: $6620-6644 \mathrm{~cm}^{-1}$ in the present case) by species in a spectroscopic cell working at low pressure (10 Torr). A sonic probe was used to couple the reactor to the cell in order to have a sufficient pressure drop between the reactor working at 800 Torr and the cell. Absorption lines used for the quantification of formaldehyde, water and hydrogen peroxide are the same as those used in [13]. The average relative uncertainty in mole fraction was estimated to be $\pm 15 \%$ depending on the absorption line used for the quantification and the concentration of the species. Absorption lines and cross sections used for the quantification of formaldehyde, water and hydrogen peroxide are given as Supplementary Material.

\section{Experimental results}

Experiments were carried out over the temperature range $475-1100 \mathrm{~K}$ (with a step of $25 \mathrm{~K}$ ), at a residence time of $2 \mathrm{~s}$, a pressure of $106.7 \mathrm{kPa}$, an inlet fuel mole fraction of 0.005 and at three equivalence ratios $(0.25,1$ and 2$)$.

Fuel mole fraction profiles are displayed in Figure 1 for the three equivalence ratios investigated. As for $n$-alkanes, the leaner the mixture, the larger the reactivity between 550 and $800 \mathrm{~K}$ (with the inlet fuel mole fraction kept constant for all $\varphi$ ). A maximum conversion of $45 \%$ is reached under rich conditions in the range 600-625 $\mathrm{K}$. The maximum conversion reaches 
about $75 \%$ in the same range for the stoichiometric and lean conditions. Hexanal displays a notable reactivity during its oxidation under the conditions of this study. This is demonstrated by some reactant conversion observed at temperatures as low as $475 \mathrm{~K}$ under lean conditions. Mole fraction profiles show a strong negative temperature coefficient (NTC) behavior but a significant reactivity can still be spotted at the end of the NTC even under rich conditions.
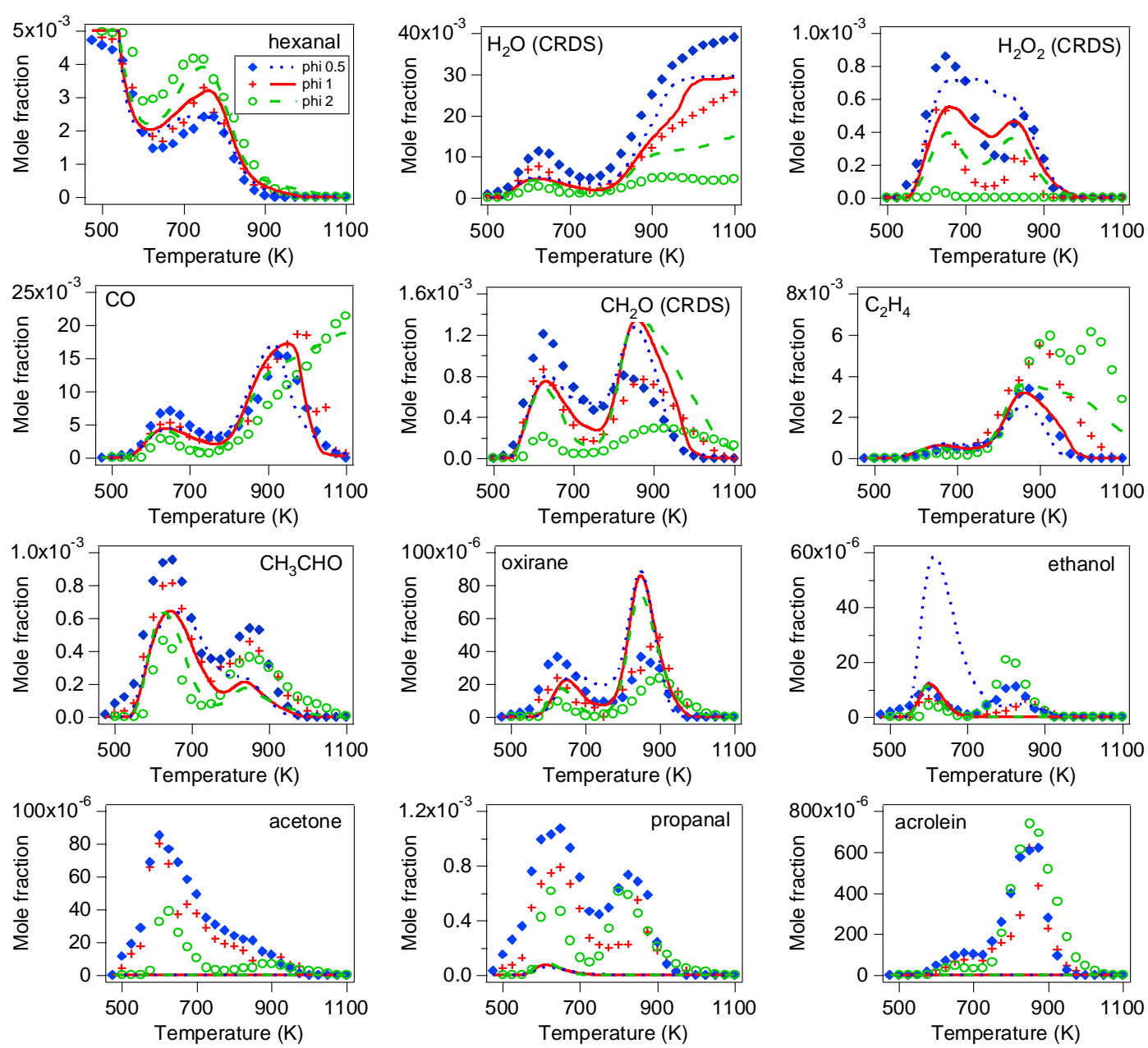

Figure 1: Mole fractions of the fuel and reaction products (up to $\mathrm{C}_{3}$ ) obtained in the oxidation of hexanal $\left(\tau=2 \mathrm{~s}, \mathrm{P}=106.7 \mathrm{kPa}, x_{\text {fuel }}^{\text {inlet }}=0.005\right)$. Symbols are for experiments and lines for simulation.

Many reaction products were detected during the oxidation of hexanal (see Figures 1 and 2 and Figure S1 in Supplementary Material). Reaction products can be sorted into two groups. The first group includes species which are usually observed during oxidation studies: $\mathrm{CO}, \mathrm{CO}_{2}$, water, $\mathrm{H}_{2} \mathrm{O}_{2}$, small $\mathrm{C}_{1}-\mathrm{C}_{3}$ hydrocarbons, aldehydes and ketones. The second group concerns specific low-temperature oxidation products. As for $n$-alkanes, some of these specific products had the same skeleton as the reactant: 5-ethyl-dihydrofuranone ( $\gamma$-caprolactone), 6-methyltetrahydropyranone ( $\delta$-caprolactone) and hexanoic acid (see Figure 3 for structures). But here it should be noted that some $\mathrm{C}_{5}$ low-temperature oxidation products with important mole fractions were also observed: 2-methyl-tetrahydrofuran and pentanal. As discussed later, these species are formed from reactions of the $n$-pentyl radical obtained from the $\alpha$-scission of the hexanoyl radical. A particularity of these $\mathrm{C}_{5}-\mathrm{C}_{6}$ low-temperature products is that their mole fraction profiles exhibit only one large peak whereas two peaks with a NTC area in between (one at low- and one at high-temperature) are observed for smaller species. 

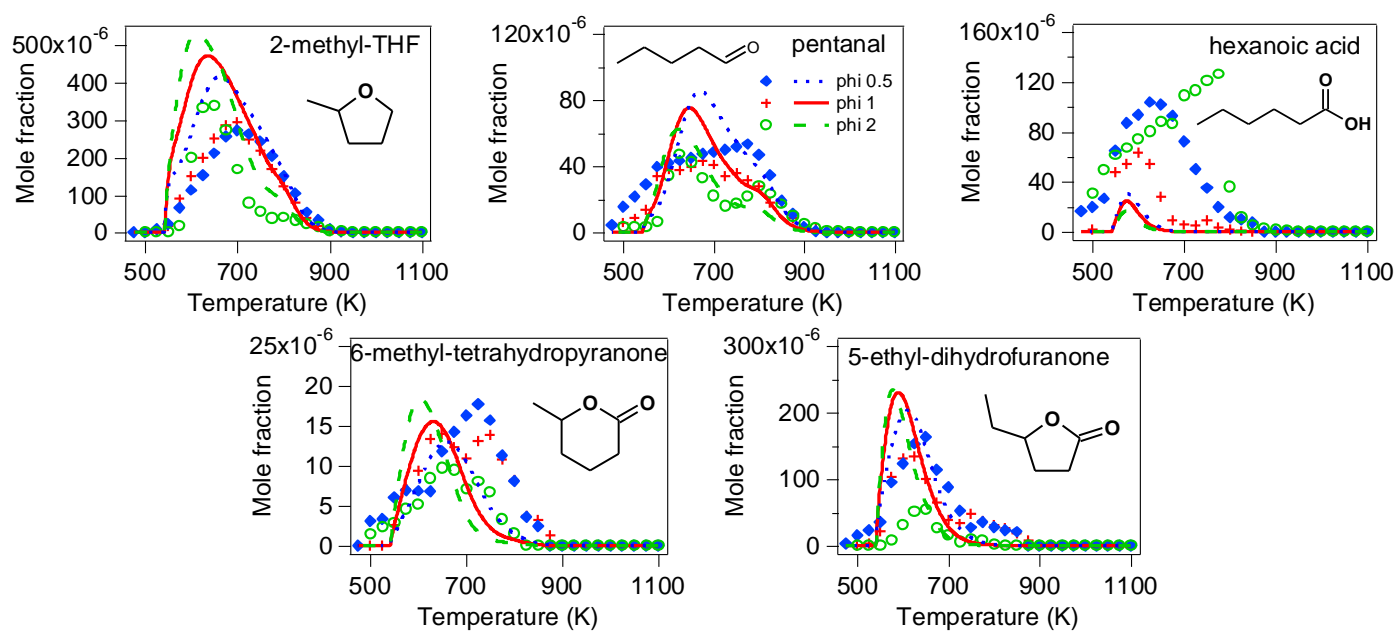

Figure 2: Mole fractions of $\mathrm{C}_{5}-\mathrm{C}_{6}$ specific low-temperature reaction products obtained in the oxidation of hexanal $\left(\tau=2 \mathrm{~s}, \mathrm{P}=106.7 \mathrm{kPa}, x_{\text {fuel }}^{\text {inlet }}=0.005\right)$. Symbols are for experiments and lines for simulation.

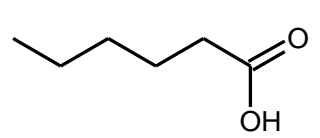

hexanoic acid

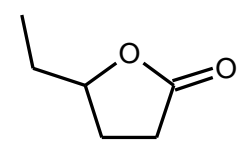

$\gamma$-caprolactone<smiles>CC1CCCC(=O)O1</smiles>

$\delta$-caprolactone

Figure 3: Structures of the $\mathrm{C}_{6}$ specific low-temperature products observed in the oxidation of hexanal.

The effect of the equivalence ratio differs from one species to another. An interesting point is that the formation of some oxygenated species is favored at low-temperature under rich conditions whereas the opposite trend is observed for most products as shown in Figure 2. This unusual promoting effect of $\varphi$ under rich conditions is clearly visible for 2-methyltetrahydrofuran. This particularity is due to the reaction of decomposition of the hexanoyl radical (yielding to the $n$-pentyl radical which is the precursor of 2-methyl-tetrahydrofuran) which is favored under rich conditions to the detriment of the reaction of addition to $\mathrm{O}_{2}$. The formation routes of acid hexanoic are likely complex as suggested by the mole fraction profiles obtained in experiments. The formation of this species seems to be favored in the NTC region under rich conditions with no obvious explanation. Another point is the sensitivity of the mole fractions of hydrogen peroxide and formaldehyde to the equivalence ratio. The maximum mole fraction of hydrogen peroxide at low-temperature obtained under rich conditions is one order of magnitude lower than that measured under lean conditions. For formaldehyde, the maximum mole fraction under lean conditions is 4 times larger than that under rich conditions.

The reactivity of hexanal was compared with that of two fuels including the same number of carbon atoms but belonging to different fuel families: 1-hexanol and $n$-hexane. Experimental fuel mole fractions obtained at $\varphi=1$ are displayed in Figure 4 . Studies were performed under the same JSR and mixture conditions. Large differences were observed between the reactivity of the three fuels. Some reactivity can already be spotted from $525 \mathrm{~K}$ for hexanal, whereas the start of the fuel consumption can only be observed from $600 \mathrm{~K}$ and $625 \mathrm{~K}$ for $n$-hexane and 1-hexanol, respectively. The most reactive species at $625 \mathrm{~K}$ was hexanal (with $70 \%$ of conversion). It was followed by $n$-hexane (conversion of $40 \%$ ) and 1-hexanol (conversion of 15\%). Another major 
difference was the absence of NTC behavior for 1-hexanol, whereas a well-marked one was observed for both hexanal and $n$-hexane. As $n$-hexane was less reactive than hexanal, the conversion was very low in the range $725-800 \mathrm{~K}$. Note however that above $750 \mathrm{~K}, 1$-hexanol was more reactive than the two other fuels.

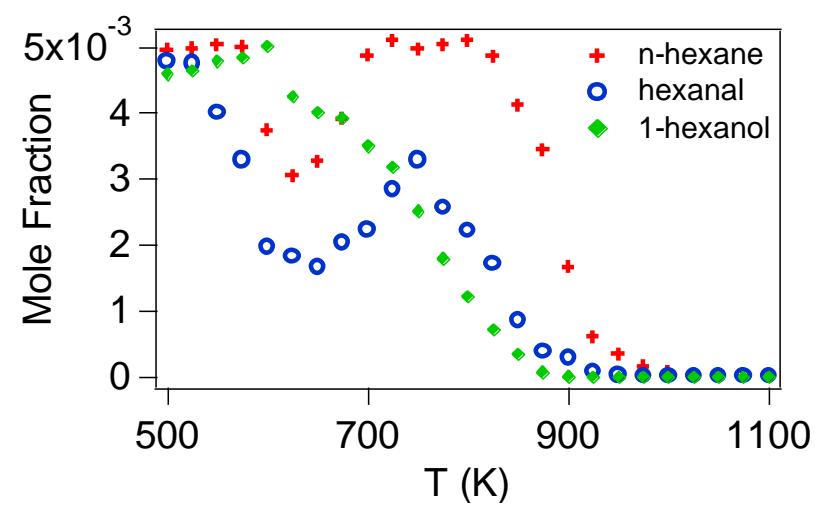

Figure 4: Comparison of experimental fuel mole fractions obtained in the oxidation of $n$-hexane, hexanal and 1-hexanol under the same conditions $(\varphi=1)$.

This comparison highlights the significant effect of the presence of an oxygen atom in the fuel on the reactivity of alcohols and aldehydes, even if the two oxygenated fuels react in very different ways. This reactivity notably depends on how the oxygen atom is linked to the rest of the molecule. An important result is that $n$-alkanes cannot be used as surrogates for long chain alcohols and aldehydes (whereas they have a reactivity somewhat similar to that of methyl esters [14]).

\section{Modeling}

A detailed kinetic model was developed for the oxidation of hexanal. The model was generated using software Exgas. Exgas is a tool for the automatic generation of detailed kinetic models for the oxidation of fuel components as alkanes [15], saturated esters [16] and alkyl-aromatics [17]. A virtual blend of hexanal and $n$-pentane was considered during the generation to better account for the chemistry of the $n$-pentyl radical which played an important role in the oxidation of hexanal as suggested by experimental observations. In addition to a $\mathrm{C}_{0}-\mathrm{C}_{2}$ reaction base including pressure dependent reactions, the mechanism generated by Exgas for hexanal and $n$ pentane considers all the primary and secondary reaction classes usually considered during alkane oxidation [15]. This involves especially all the reactions of the alkylperoxy radicals, including isomerizations and formation of cyclic ethers and ketohydroperoxides.

However some reactions were missing in the generated model because of some new reactions due to the presence of the aldehyde group. As an example, the only reaction written for the hexanoyl radical (R29C6H110K) was the decomposition through $\alpha$-scission to CO and $n$-pentyl radical. The low-temperature oxidation chemistry of the hexanoyl radical was not considered in the generated model and the missing chemistry set was written by hand (see Figure S2 in Supplementary Material). The new reaction considered for the hexanoyl radical was the addition of this radical to $\mathrm{O}_{2}$ leading to the formation of a peroxy radical. Reactions considered for this peroxy radical were isomerizations to hydroperoxy-alkyl radicals and a specific reaction to 
aldehydes which is the combination with $\mathrm{HO}_{2}$ radicals to form hexanoic acid or peroxy hexanoic acid as proposed by Le Crâne et al. [18] (see Figure 5). Kinetic constants for H-atom abstractions by $\mathrm{OH}$ and $\mathrm{HO}_{2}$ are those from Exgas except for the specific $\mathrm{H}$-atoms of the aldehyde group and those in the beta position which were taken from the study by Pelucchi et al. [8]. A comparison of Exgas kinetic data with literature ones is given in Supplementary Material. It shows a good agreement between Veloo et al. [11] and Exgas data. The addition of $\mathrm{OH}$ radicals to the carbon atom of the carbonyl function has also been written but this does not contribute significantly to the formation of hexanoic acid. Reactions considered for hydroperoxy-alkyl radicals are the second addition to $\mathrm{O}_{2}$ and reactions of decomposition to cyclic ethers or unsaturated species. Note that these reactions are responsible for the formation of $\gamma$-caprolactone and $\delta$-caprolactone that were detected in experiments. Kinetic parameters used for the added low-temperature reactions are those already used in Exgas for alkanes [15] (except the reactions for hexanoic acid or peroxy hexanoic acid, for which the kinetic parameters are those proposed by Le Crâne et al. [18]). The activation energy of the reaction of $\alpha$-scission of the hexanoyl radical was increased from 17.2 to $20.0 \mathrm{kcal} . \mathrm{mol}^{-1}$ to obtain a better agreement between computed and experimental data. Note that the kinetic constant proposed in [8] for the $\alpha$-scission was tested but appeared to be too fast. The model described in this paper contains 482 species involved in 2590 reactions. It is given under Chemkin Format in Supplementary Material. Simulations were performed using the PSR module in the Chemkin II package [19].

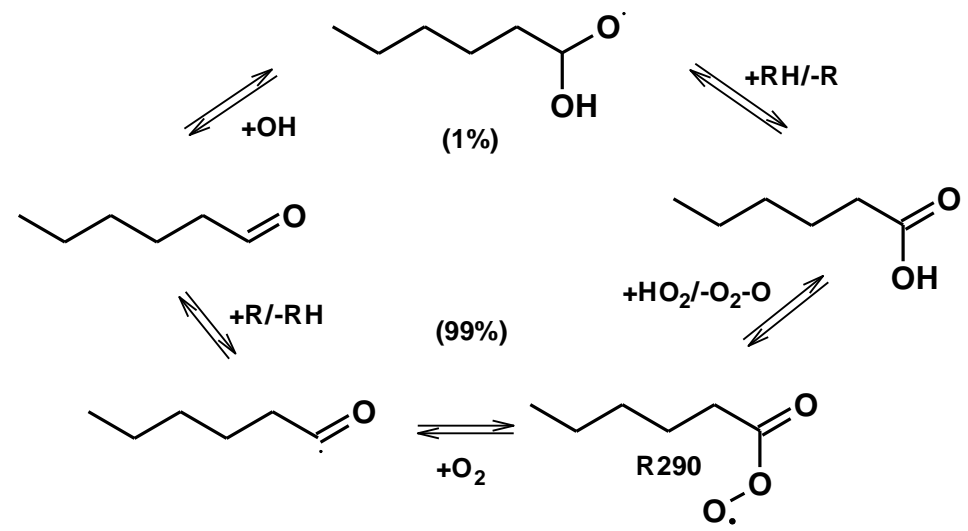

Figure 5: Formation routes of hexanoic acid at $625 \mathrm{~K}$. Percentages correspond to relative formation flux of hexanoic acid.

\section{Discussion}

Mole fractions computed using the model are displayed in Figures 1 and 2. As far as the reactivity is concerned, the model predicts well the NTC behavior at all equivalence ratios, but simulations over-predict the fuel consumption under rich conditions between 600 and $700 \mathrm{~K}$. The reactivity over-estimation under rich conditions causes an over-prediction of product mole fractions between 600 and $700 \mathrm{~K}$. Under lean and stoichiometric conditions, the agreement is overall satisfactory for water, $\mathrm{CO}$, formaldehyde, acetaldehyde. The agreement is also acceptable for specific $\mathrm{C}_{5}$ and $\mathrm{C}_{6}$ products, but the discrepancies which can be spotted in the graphs of Figure 2 show that refinements are still needed to better account for the chemistry of the oxidation of hexanal. 
A kinetic analysis of the model was performed to identify sensitive reactions and to propose possible improvements of the oxidation chemistry of large aldehydes. According to the rate of production analysis performed at $625 \mathrm{~K}$ for the stoichiometric mixture (see Figures S3 and S4), the fuel is mainly consumed via $\mathrm{H}$-atom abstractions (with $\mathrm{OH}$ radicals). Note that the formation of the hexanoyl radical (R29 in the model) is favored due to the lower C-H bond energy (35\% of the consumption of hexanal) but that other $\mathrm{H}$-atom abstractions are not negligible (between 5 and $24 \%$ according to the nature of the abstracted $\mathrm{H}$-atom). An important point is that all radicals but one (that with the radical center in position 3) finally isomerize yielding hexanoyl radical (these isomerizations preferentially involve five and six membered ring transition states) and that overall $80 \%$ of the consumed fuel lead to this $\mathrm{C}_{5} \mathrm{H}_{9} \mathrm{CO}$ radical. The hexanoyl radical reacts through two competitive pathways: 1 ) it decomposes via an $\alpha$-scission to the $n$-pentyl radical and $\mathrm{CO}(67 \%), 2)$ it adds to $\mathrm{O}_{2}$ to form a peroxy-aldehyde radical (33\%). Veloo et al. observed an opposite ratio for the $\alpha$-scission and the addition to $\mathrm{O}_{2}$ of the butanoyl radical in their butanal study [11]. A test with their kinetic constants led to large discrepancies in the prediction of the mole fractions of $\mathrm{C}_{5}$ and $\mathrm{C}_{6}$ low-temperature oxidation products. Then the $n$ pentyl radical obtained via the $\alpha$-scission undergo the typical low-temperature oxidation chemistry of alkanes (which is included in the chemistry of oxidation of $n$-pentane virtually considered in the list of reactants for the automatic generation). This chemistry is responsible for the formation of 2-methyl-tetrahydrufuran and pentanal. The peroxy-aldehyde radical (R290 in the flux diagrams in Figure S3) mainly reacts via isomerizations to yield new hydroperoxy radicals which can add to $\mathrm{O}_{2}$ for the branching or decompose to cyclic ethers (such as the two caprolactones observed in experiments). The second consumption route for the peroxyaldehyde radical is the combination with $\mathrm{HO}_{2}$ radical yielding hexanoic acid and peracid (1.7 and $6.5 \%$, respectively). Hexanoic acid is mainly formed from the reaction of combination of R290 with $\mathrm{HO}_{2}$ (99\%), whereas the more direct route starting with the addition of $\mathrm{OH}$ to the carbonyl group represents less than $1 \%$ of the formation of this acid.

Figure 6 displays the sensitivity analysis on the fuel mole fraction performed at $625 \mathrm{~K}$ and $\varphi=1$ (sensitivity analyses at the three $\varphi$ are given in Supplementary Material). The sensitive reactions promoting the reactivity are $\mathrm{H}$-atom abstractions (especially that abstracting the $\mathrm{H}$-atom of the carbonyl group forming the hexanoyl radical) and the decomposition of the hexanoyl radical to $\mathrm{CO}$ and $n$-pentyl radical. $\mathrm{H}$-atom abstractions are likely not responsible for the discrepancies observed at low-temperature for $\varphi=0.5$ and 2 as their effect is similar whatever $\varphi$. The most sensitive reactions inhibiting the reactivity are concurrent reactions consuming $\mathrm{OH}$ radicals such as $\mathrm{H}$-atom abstractions involving acetaldehyde and formaldehyde, the reaction of addition of the hexanoyl radical to $\mathrm{O}_{2}$ (forming the peroxy radical $\mathrm{R} 290$ ) which competes with its

decomposition, and the isomerization of R290 to R292 (o) This last reaction is important because R292 decomposes to the $\gamma$-caprolactone $+\mathrm{OH}$ (an inhibitive propagation pathway). Its inhibitive effect is more important under lean conditions. Under rich conditions, reactions involved in the low-temperature chemistry of the $n$-pentyl radical are likely responsible for the observed discrepancies (they have a promoting effect). The main lesson to be learnt from the kinetic analysis is that a more accurate set of kinetic parameters would be required for carbonyl containing species to better account for the chemistry of oxidation of long chain aldehydes. 


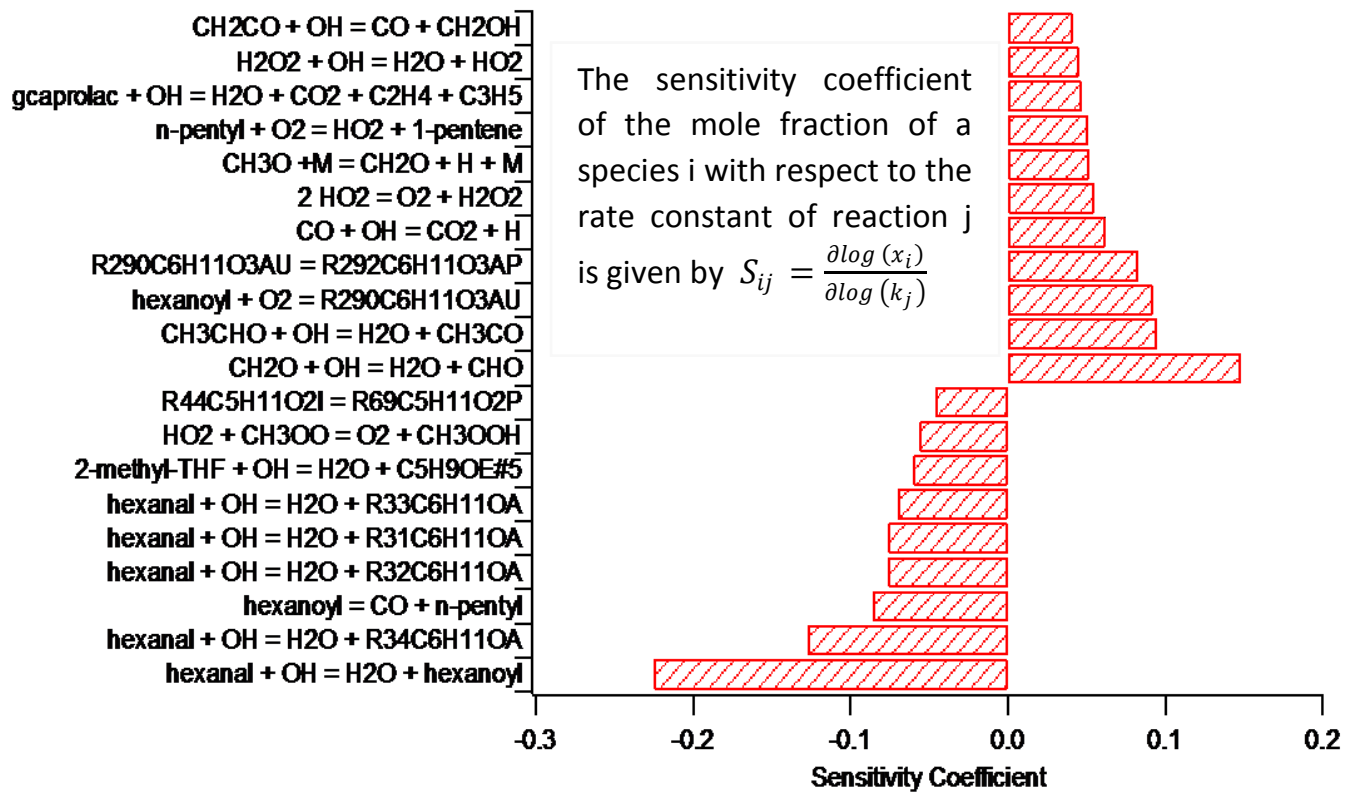

Figure 6: Sensitivity analysis on the fuel mole fraction performed at $625 \mathrm{~K}$ (see Figure S2 in Supplementary Material for the structures of the species).

\section{Conclusion}

The study of the oxidation of hexanal was performed in a jet-stirred reactor with reaction product measured using gas chromatography and continuous wave cavity ring-down spectroscopy. This study highlighted the high reactivity of long chain aldehydes compared to their alkane homologues, as well as the formation of specific reaction products with the same skeleton as the reactant (hexanoic acid, $\gamma$ - and $\delta$-caprolactones), but also $\mathrm{C}_{5}$ ones coming from the $n$-pentyl radical oxidation (e.g., 2-methyl-tetrahydrofuran). Modeling efforts have been carried out to develop a mechanism accounting for the specific chemistry of the oxidation of hexanal with acceptable agreement for the reactivity and main product mole fractions. The kinetic analysis performed at $625 \mathrm{~K}$ highlighted the determinant role played by the hexanoyl radical during the oxidation of hexanal. Because of preferential isomerizations due to the lower $\mathrm{C}-\mathrm{H}$ bond energy, almost $80 \%$ of the fuel is transformed to the hexanoyl radical. The two main consumption pathways of the hexanoyl radical (decomposition via an $\alpha$-scission and addition to $\mathrm{O}_{2}$ ) are then responsible for the selectivity of $\mathrm{C}_{5}$ and $\mathrm{C}_{6}$ specific low-temperature reaction products which were experimentally observed. More experimental and theoretical studies on the low-temperature oxidation of aldehydes are necessary to improve the chemistry in detailed kinetic models.

\section{Supplementary Materials}

- Mechanism file (Chemkin II format)

- Supplementary file (word file) 


\section{References}

[1]F. Battin-Leclerc, A. Rodriguez, B. Husson, et al., J. Phys. Chem. A, 118 (2014), pp. 673-683

[2]C. Togbé, P. Dagaut, A. Mzé-Ahmed, P. Diévart, F. Halter, F. Foucher, Energy Fuels, 24 (2010), pp. 5859-5875

[3]T. Hosoya, H. Kawamoto, S. Saka, J. Anal. Appl. Pyrolysis, 80 (2007), pp. 118-125

[4]A. Lifshitz, C. Tamburu, A. Suslensky, J. Phys. Chem., 94 (1990), pp. 2966-2972

[5]D.F. Davidson, S.C. Ranganath, K.-Y. Lam, M. Liaw, Z. Hong, R.K. Hanson, J. Propuls. Power, 26 (2010), 280-287

[6]B. Akih-Kumgeh, J.M. Bergthorson, Combust. Flame, 158 (2011), 1877-1889

[7]J. Zhang, L. Pan, J. Mo, J. Gong, Z. Huang, C.K. Law, Combust. Flame, 160 (2013), 1541-1549

[8]M. Pelucchi, K.P. Somers, K. Yasunaga, et al., Combust. Flame, 162 (2015), 265-286

[9]T. Kasper, U. Struckmeier, P. Oßwald, K. Kohse-Höinghaus,Proc. Combust. Inst., 32 (2009), 1285-1292

[10]P.S. Veloo, P. Dagaut, C. Togbe, et al., Proc. Combust. Inst., 34 (2013), 599-606

[11]P.S. Veloo, P. Dagaut, C. Togbé, et al., Combust. Flame, 160 (2013), 1609-1626

[12]0. Herbinet, G. Dayma, Clean Combustion, F. Battin-Leclerc, J.M. Simmie, E. Blurock (Eds.)Springer London (2013), 183-210

[13]A. Rodriguez, O. Frottier, O. Herbinet, et al., J. Phys. Chem. A, 119 (2015), 7905-7923

[14]C.K. Westbrook, C.V. Naik, O. Herbinet, et al., Combust. Flame, 158 (2011), 742-755

[15]J. Biet, M.H. Hakka, V. Warth, P.-A. Glaude, F. Battin-Leclerc, Energy Fuels, 22 (2008), 22582269

[16]P.A. Glaude, O. Herbinet, S. Bax, J. Biet, V. Warth, F. Battin-Leclerc, Combust. Flame, 157 (2010), 2035-2050

[17] F. Battin-Leclerc, V. Warth, R. Bounaceur, B. Husson, O. Herbinet, P.-A. Glaude, Proc. Combust. Inst., 35 (2015), 349-356

[18]J.-P. Le Crâne, M.-T. Rayez, J.-C. Rayez, E. Villenave, Phys. Chem. Chem. Phys., 8 (2006), 21632171

[19]R.J. Kee, F.M. Ruplay, J.A. Miller, Sandia Laboratories Report S 89-8009B, 1993. 